Revue européenne des sciences sociales

European Journal of Social Sciences

XLIV-134 | 2006

Quel(s) défi(s) pour les sciences sociales à l'heure de la mondialisation?

\title{
Évolution de la société : quel avenir pour les émotions?
}

\section{Klaus Scherer}

\section{(2) OpenEdition Journals}

Édition électronique

URL : http://journals.openedition.org/ress/314

DOI : $10.4000 /$ ress.314

ISSN : 1663-4446

Éditeur

Librairie Droz

Édition imprimée

Date de publication : 1 juin 2006

Pagination : 277-289

ISBN : 9-782-600-01095-5

ISSN : 0048-8046

Référence électronique

Klaus Scherer, «Évolution de la société : quel avenir pour les émotions ? 》, Revue européenne des

sciences sociales [En ligne], XLIV-134 | 2006, mis en ligne le 15 octobre 2009, consulté le 01 mai 2019 URL : http://journals.openedition.org/ress/314 ; DOI : 10.4000/ress.314

Ce document a été généré automatiquement le 1 mai 2019.

(c) Librairie Droz 


\title{
Évolution de la société : quel avenir pour les émotions ? ${ }^{1}$
}

\author{
Klaus Scherer
}

\section{La dimension sociale des émotions}

1 Les émotions sont devenues un sujet très apprécié dans la recherche et les publications se font nombreuses. Par ailleurs, la tendance actuelle de privilégier le cerveau comme lieu d'investigation psychologique par excellence entraîne un intérêt croissant pour les études essayant de déterminer la localisation cérébrale des phénomènes émotionnels. Il s'agit d'une entreprise louable, mais le danger de négliger la nature essentiellement sociale des émotions subsiste. De plus, les émotions ont un passé - comme démontré par le rôle important que certains climats émotionnels spécifiques ont joué en certaines périodes et dans certaines cultures (voir par exemple, Delumeau, 1978; Lepenies, 1969; Stearns \& Stearns, 1986, 1988) - tout comme un avenir. Du fait d'un changement social toujours plus rapide, on pourrait imaginer que la genèse, l'expérience et la régulation des émotions changeront, en même temps que leur fonction sociale. Cette contribution se focalise sur cette question. En particulier, seront abordés dans ce qui suit : 1) l'effet de changements normatifs sur les émotions autoréflexives, telles que la honte et la culpabilité; 2) les effets sur le plan émotionnel de l'exposition aux mass media, et 3) l'effet des innovations technologiques sur la régulation des émotions.

\section{Les conséquences de changements sociaux sur la genèse des émotions}

2 La honte et la culpabilité se classent parmi les émotions qui se prêtent le plus difficilement à l'analyse scientifique. Elles ne figurent souvent pas sur la liste des émotions fondamentales, et les psychologues et psychanalystes ont eu beaucoup de peine à se mettre d'accord sur leurs différences (Gaulejac, 1996; Lewis, 1992; Lynd, 1970; Piers, 1971). Pour les théoriciens de l'appraisal, la honte et la culpabilité se produisent quand 
une personne évalue son comportement (dans ce cas généralement, l'événement qui déclenche l'émotion) comme incompatible soit avec son idéal de soi, soit avec les normes et valeurs socioculturelles. Ainsi, je ressentirais de la honte si l'on me voyait jeter des ordures dans la rue afin de gagner du temps - alors que je me considère par ailleurs comme une personne respectueuse de l'environnement. De même, je devrais ressentir une certaine culpabilité si j'étais quelqu'un de très croyant et que je trompais mon partenaire. Utilisant la honte comme exemple, Borg, Staufenbiel \& Scherer (1988) ont essayé de spécifier les conditions sous lesquelles le non-respect des valeurs produirait inéluctablement des sentiments d'embarras ou de honte.

3 Etant donné la relation étroite entre la honte/la culpabilité et les valeurs socioculturelles/l'idéal de soi, la nature et l'incidence de ces émotions devraient varier considérablement selon le changement des normes dominantes, des valeurs et des idéaux propres à chacun dans une culture particulière à un moment donné. Il existe actuellement certaines données empiriques qui confirment cette hypothèse.

Dans une étude interculturelle conduite dans 37 pays des 5 continents, Wallbott et Scherer (1995) ont trouvé relativement peu de différences entre honte et culpabilité au sein des cultures dites individualistes (cultures dont la structure sociale accorde beaucoup d'importance aux droits et intérêts de chacun). Tant pour la honte que pour la culpabilité, le comportement à l'origine de l'émotion était considéré comme extrêmement immoral. $\mathrm{Au}$ contraire, dans les cultures dites collectivistes (sociétés dont la priorité est axée sur les intérêts familiaux ainsi que sur ceux des groupes sociaux), les sentiments de culpabilité étaient provoqués plus fréquemment par des actes jugés bien plus immoraux que ne l'étaient ceux qui induisaient la honte. Les données, en accord avec ces divergences d'appréciation, indiquaient aussi des différences significatives pour d'autres composants de ces deux émotions. Les expériences de honte dans les cultures collectivistes étaient intenses mais courtes, sans conséquences majeures. En comparaison, dans les cultures individualistes, les réactions comportementales typiques des expériences de honte étaient hautement comparables à celles des expériences de culpabilité (comportant des effets à long terme sur l'évaluation personnelle). Cette étude fournit donc la preuve que les différences culturelles concernant l'importance de valeurs distinctes peuvent affecter la genèse des émotions ainsi que les caractéristiques de leur expression.

5 Les structures de croyance représentent également une autre source potentielle de différence entre les cultures dans l'évaluation cognitive des événements. Selon l'étude interculturelle mentionnée précédemment, les participants issus des pays africains avaient uniformément caractérisé les événements induisant une émotion comme plus immoraux et plus systématiquement causés par des agents externes que les participants provenant d'autres cultures. Un système de croyance basé sur la magie et la sorcellerie, encore répandu dans ces pays, pourrait expliquer en partie ces données. En effet, cette tradition privilégie le fait d'incriminer et de blâmer moralement des agents externes présumés artificiels pour les événements négatifs vécus (Scherer, 1997). En revanche, les participants des pays d'Amérique Latine et du Sud tendaient à juger les événements provoquant les émotions systématiquement plus bas sur la dimension d'immoralité que des participants des autres pays étudiés. Le niveau d'urbanisation étant beaucoup plus élevé dans les pays latino et sud-américains étudiés (la plupart des participants vivant dans de grandes villes), nous avons spéculé que l'exposition plus fréquente au nonrespect des normes dans les grands secteurs urbains pourrait expliquer ce résultat. Dans les deux cas, ces interprétations restent spéculatives. Pourtant, ces données suggèrent 
que les facteurs socioculturels peuvent fortement affecter les dimensions d'évaluation impliquées dans la genèse de la honte et de la culpabilité.

Ces exemples proposent qu'une évolution sociale continue - impliquant des changements sur le système de valeurs, de normes ainsi que sur les idéaux de soi propres à chaque individu - devrait avoir un grand impact sur l'expérience de la honte et de la culpabilité. Tandis qu'il n'existe que peu de données empiriques à ce sujet, beaucoup d'éléments du sens commun indiquent que les contraintes normatives s'affaiblissent et que les valeurs perdent leurs fonctions de contrôle, pour beaucoup de sociétés occidentales avec un niveau de vie élevé du moins. Le symptôme souvent évoqué concerne une augmentation des comportements indésirables ou immoraux aussi bien que le non-respect des règles qui demeure impuni. Alors que par le passé on ne se serait pas mal conduit en raison de scrupules moraux(ou par crainte de la honte et de la culpabilité conséquentes), on s'engage maintenant dans de tels comportements s'il n'y a aucune crainte de punition. Les signes actuellement évoqués par une grande partie de la critique sociale, pour n'en nommer que quelques-uns, sont l'augmentation du vandalisme, de la permissivité sexuelle, de la petite délinquance, des crimes économiques, des mauvaises manières, de la violence, etc.

7 Si cette analyse est correcte, on pourrait présumer que l'atténuation croissante des valeurs pro-sociales et des standards propres à chacun diminuera l'incidence de la honte et de la culpabilité dans la société moderne. Une telle tendance aurait assurément des effets importants sur le fonctionnement de la société. Les émotions autoréflexives, en particulier la honte et la culpabilité, ont souvent été considérées comme des émotions socialisantes, c'est-à-dire comme éléments de l'internalisation des valeurs sociales et des normes morales. Elles remplissent de ce fait une fonction importante dans le contrôle social (Scheff, 1990). Du fait que le mécanisme normal du contrôle social (interdictions explicites, surveillance et punition) est extrêmement coûteux et inefficace, la plupart des sociétés ont compté sur les mécanismes internes de contrôle. Ces derniers sont intégrés durant le processus de socialisation de l'enfant et fonctionnent à travers l'assimilation de la honte et de la culpabilité. En effet, une des techniques standard de socialisation a été de susciter des sentiments de honte ou de culpabilité en réponse à une transgression ( $\mathrm{Tu}$ n'as pas honte? » ou « Honte à toi !»). De telles techniques dites de shaming semblent être en train de disparaître. En effet, lorsqu'on y a recours, elles ne provoquent souvent que la dérision. Si la tendance à évaluer moralement le comportement s'affaiblit, on pourrait également imaginer que la fréquence et l'intensité de certaines émotions, telles que le mépris, diminueront. Réciproquement, si l'égoïsme est en augmentation, comme le suggèrent de nombreux critiques sociaux, les manifestations de la colère - due à la frustration - devraient augmenter.

8 En imaginant que la honte et la culpabilité perdent de leur importance ou disparaissent en tant qu'agents de socialisation, il serait intéressant de voir si d'autres émotions prendraient leur place. Durant l'âge d'or de l'anti-autoritarisme, on a souvent clamé que la honte et la culpabilité étaient des émotions répressives, reflétant les principes injustifiés arbitrairement imposés par des structures autoritaires. La revendication était que le respect des intérêts et des droits de chacun sont des principes plus démocratiques qui devraient régir les interactions sociales. Selon cette idéologie, ce ne devrait pas être l'anticipation de la honte qui préviendrait les comportements anti-sociaux, mais la prise de conscience qu'en se comportant ainsi cela porterait préjudice à une tierce personne ou à la société. L'idée sous-jacente semble être que l'on puisse remplacer la honte et la 
culpabilité par l'empathie. Toutefois, plutôt que de supposer que l'empathie remplace la honte et la culpabilité, on pourrait s'inquiéter du fait que cette capacité s'affaiblisse également. L'énorme impact du livre très populaire de Goleman (1995) sur l'intelligence émotionnelle, prônant des mesures éducatives visant à améliorer les capacités émotionnelles des enfants et des adolescents, montre l'étendue du problème. Il est intéressant de relever que les cours de compétences émotionnelles sont de plus en plus souvent intégrés dans les programmes d'études de beaucoup de pays. Malheureusement, il semble y avoir une grande variabilité individuelle dans la propension à montrer de l'empathie envers autrui (Bischof-Köhler, 1991).

\section{Les effets des émotions médiatiques}

Une grande partie des émotions est occasionnée par l'exposition à des objets ou événements, tels qu'accidents, menaces, succès ou échecs, actes de tiers, etc. Une autre catégorie d'émotions fait suite à des événements concernant directement la personne en raison de leur signification par rapport à ses besoins, valeurs ou buts importants. Les mécanismes sous-tendant la génération de telles émotions, constitués principalement par une série d'évaluations cognitives, commencent à être bien connus (Ellsworth \& Scherer, 2003; Roseman \& Smith, 2001; Scherer, 2001). Le processus sous-jacent est illustré dans la partie supérieure du Graphique 1 (cadres gris et flèches solides). En revanche, il reste encore une classe d'émotions dont la genèse est moins claire. Il s'agit des émotions qui sont déclenchées par le simple fait d'observer quelqu'un d'autre vivre un état émotionnel fort, un phénomène que j'ai proposé d'appeler une commotion en analogie avec le terme compassion (Scherer, 1998). La partie inférieure du Graphique 1 illustre trois mécanismes qui pourraient expliquer la production de telles commotions : 1) l'induction à partir de la situation ou de l'événement observé (basée également sur un processus d'évaluation et impliquant que l'événement ait une signification comparable pour l'observateur), 2) l'empathie comprise comme la compassion envers l'individu qui est en train d'expérimenter l'émotion déclenchée par l'événement, et 3) la contagion par les symptômes observés. Les mécanismes d'empathie et de contagion diffèrent dans le sens qu'il n'est pas nécessaire de comprendre la réaction de l'individu observé pour le second. Par exemple, l'observation d'un tremblement des membres suffirait à occasionner une imitation de ces manifestations corporelles chez l'observateur, tremblements qui à leur tour produiraient un sentiment émotionnel chez lui. Ces trois processus pourraient agir seul ou en interaction. 
Graphique 1

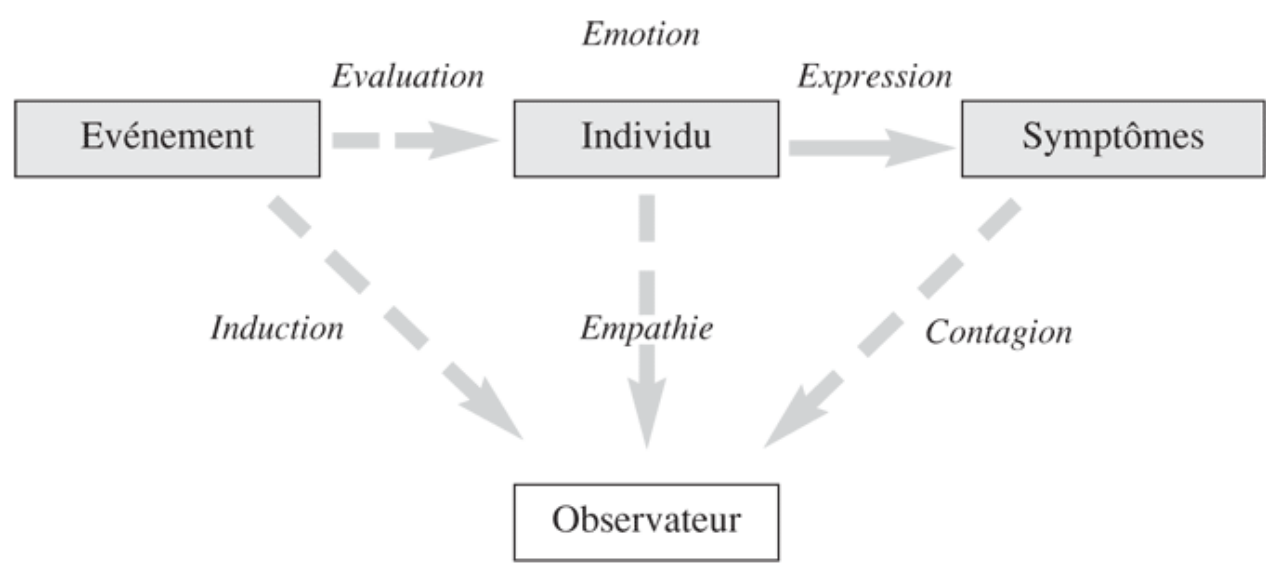

Emotion

10 En général, les émotions sont donc produites par des événements importants que nous vivons ou par l'observation des émotions expérimentées par autrui. Ceci étant, on ne devrait pas sous-estimer la fréquence des cas dans lesquels des émotions sont obtenues indirectement. Il s'agit ici notamment des émotions ressenties face à un reportage ou aux commentaires relatifs à un événement dans les médias, dans les interactions virtuelles du monde des jeux informatisés/vidéo ou encore dans les chats sur l'Internet. Lors d'une étude interculturelle comparative menée dans 8 pays européens, Scherer, Wallbott et Summerfield (1986) ont remarqué qu'environ $20 \%$ des expériences émotionnelles rapportées par les participants avaient été générées par la radio ou les nouvelles télévisées, les articles de journaux, etc. A l'époque, nous avions été étonnés par ce pourcentage plutôt élevé, mais les études ultérieures ont réaffirmé l'importance des conséquences émotionnelles de l'exposition aux médias (Cantor et Nathanson, 1996; Gleich et Grobel, 1993; Johnston et Davey, 1997; Winterhoff-Spurk, 1997). Il serait intéressant d'étudier si ce pourcentage a augmenté depuis (Wallbott et Scherer, 1986).

11 Les émotions produites par les médias diffèrent-elles de celles induites directement par des objets ou événements rencontrés dans la vie quotidienne? Une différence principale pourrait être que l'événement montré ou rapporté dans les médias est généralement d'une certaine importance pour un grand nombre de personnes, et ainsi nécessairement d'importance immédiate moindre pour chaque personne considérée individuellement. Il est probable que les préoccupations hautement personnelles, telles que le bien-être, l'amour-propre ou les buts/besoins particuliers (Frijda, 1986; Scherer, 1988) soient rarement affectées par l'événement médiatique. Il s'agit plus généralement de valeurs partagées culturellement, telles que la justice ou la conformitéaux normes. En outre, puisque l'exposition aux médias et l'état émotionnel résultant peuvent potentiellement être partagés avec d'autres personnes, cela rend une comparaison, une amplification, voire une contagion sociale possible. Si, en effet, on admet que les émotions médiatisées sont potentiellement une classe séparée des émotions dites directes et que leur nombre augmente dans les sociétés modernes, il est de grand intérêt d'analyser leur nature plus en détails.

Ci-dessus, nous avons discuté de laproductiondes émotions par le contenu des médias. Cependant, il est potentiellement plus intéressant de s'interroger sur la forme 
émotionnelle de ce contenu. Tandis qu'il n'existe pratiquement aucune donnée empirique sur le sujet, on a la nette impression que les mass media, particulièrement la télévision, utilisent de plus en plus un matériel fortement émotionnel pour garder l'intérêt de leur public et pour vendre leurs produits. Ainsi, il semble que l'analyse systématique des événements ait été remplacée par des interviews où l'aspect émotionnel est accentué. Par exemple, plutôt que d'analyser les sources structurales de mécontentement qui a mené à une grève, les nouvelles présenteront souvent un ouvrier fâché, exprimant son émotion. De même, la couverture dramatique de guerres et de catastrophes, n'épargnant aucun détail sanglant, est devenue courante à l'apex de l'émotion. Dans la même veine, les émissions de (télé)réalité, de jeux et le récent « Big Brother » semblent avoir comme seul but de montrer des émotions à l'écran, et de solliciter par ce biais des émotions auprès des téléspectateurs.

Graphique 2

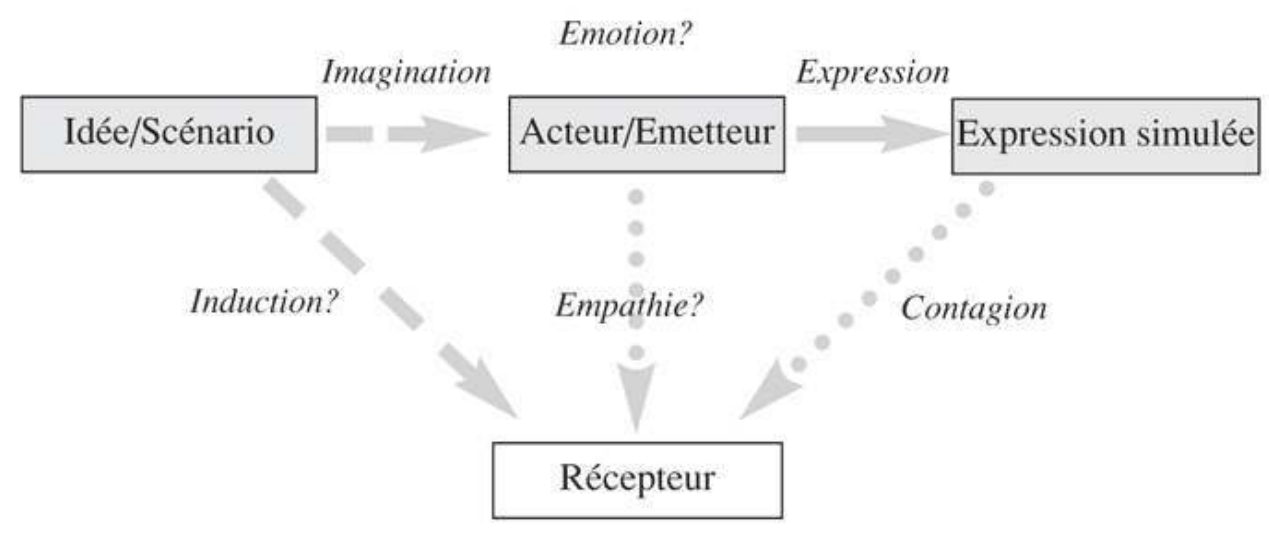

Emotion?

Qu'en est-il des effets émotionnels suscités par l'observation des émotions de tierces personnes à la TV ? On pourrait débattre du fait que le modèle représenté sur le Graphique 1 s'applique aussi à ce cas, la seule différence étant que l'observation de l'émotion émise s'effectue par l'intermédiaire de la couverture médiatique plutôt que par l'observation directe. Cependant, ceci n'est pas tout à fait vrai. Même si les médias rapportent des cas réels sans tenter de dramatiser la situation, ce qui est montré à l'écran n'est qu'un élément de l'épisode émotionnel original. Le spectateur manquera souvent d'une compréhension complète de la nature précise de l'événement qui a déclenché l'émotion. Il ne sait que très peu de chose au sujet de la personne et de ses réactions habituelles et n'a accès qu'à un bref aperçu du comportement expressif. Jusqu'ici, nous savons peu des effets émotionnels de telles émotions de réalité dans les médias (mais voir Schorr, 1995).

En revanche, les conséquences sur le plan émotionnel de l'exposition à des émotions fictives transmises par les médias ont été intensément discutées, la plupart du temps au sujet des effets de la violence sur les téléspectateurs (Anderson \& Bushman, 2002). Malheureusement, la majeure partie de cette discussion a porté sur le statut d'évidence de l'effet déclencheur de la violence des médias, en particulier chez les adolescents. Tandis que la majorité des experts en sciences sociales considèrent ce lien entre médias et violence comme avéré et ont demandé une réglementation politique (par exemple, par un lobbying important de la part de l'Association des Psychologues des Etats-Unis), 
l'industrie des médias continue à nier l'existence de tels effets (voir Freedman, 2002, un travail financé par l'industrie concernée) et a su jusqu'ici éviter toute forme de réglementation de la part d'organismes de surveillance gouvernementaux.

Cependant, il n'y a eu que peu d'intérêt à mieux comprendre l'effet des manifestations émotionnelles fictives sur la réaction affective du spectateur. Le Graphique 2 reprend une tentative d'analyse du phénomène dans le cas d'observations d'émotions réelles (comme représenté sur le Graphique 1). La principale différence réside dans le fait que l'émetteur est exposé à un scénario fictif, et non à un événement réel, pour susciter son émotion. L'acteur/émetteur représente ensuite cette idée dans son esprit afin de dépeindre l'émotion souhaitée par l'expression appropriée. Selon l'école de théâtre suivi, l'acteur peut réellement tenter de simuler une évaluation réaliste de la situation; il se projette luimême entièrement dans la situation afin de vivre à travers celle-ci et produire, au moins en partie, une émotion ressentie, comme cela est le cas pour la technique Stanislavski.

Quel est l'effet de la production d'émotions sur le spectateur via l'un des trois mécanismes décrits précédemment? Bien que ce soit probablement rare, on ne peut pas exclure que le spectateur puisse se plonger dans une situation fictive afin de réellement ressentir l'émotion visée, en utilisant des techniques semblables à celles d'un acteur stanislavskien. L'explication plus classique est toutefois que les expériences émotionnelles vécues au théâtre et au cinéma soient un mélange d'induction et d'empathie. Le spectateur entre dans la peau de l'acteur, probablement par un processus d'identification ou de prise de rôle, et éprouve ses émotions par procuration.

Le mécanisme de la contagion émotionnelle a récemment gagné une acceptation croissante. Il y a maintenant un certain nombre de données qui indiquent que ce mécanisme est possible simplement sur la base d'une exposition à l'expression, sans devoir postuler des processus d'empathie ou d'identification à l'émetteur (Baudonnière, 1997; Hatfield, Caioppo et Rapson, 1993; Lakin, Jefferis, Cheng \& Chartrand, 2003). Cette hypothèse est également suggérée par la capacité de la musique à induire des émotions. Il est bien connu que certains types de musique peuvent produire des états de transe dans les sociétés traditionnelles (Scherer \& Zentner, 2001). Des mécanismes semblables peuvent être attendus pour le rock moderne et en particulier pour la musique techno. Par conséquent, il semble probable que la contagion émotionnelle fonctionne par l'intermédiaire de modèles expressifs (par exemple, les configurations faciales dans la modalité visuelle, le rythme dans la modalité auditive).

Après cette introduction générale, nous pourrions nous demander ce que ces mécanismes présagent pour le futur de l'émotion. Une possibilité est que la proportion relative des commotions à émotions augmente. Etant donné l'omniprésente exposition à des phénomènes émotionnels transmis par les médias, les (télé)spectateurs/récepteurs peuvent éprouver plus fréquemment que par le passé des commotions produites par empathie et contagion. On peut alors s'interroger sur les répercussions de tels phénomènes sur la vie émotionnelle. Une possibilité est que ces phénomènes, particulièrement quand ils sont d'intensité élevée, submergeront les capacités émotionnelles des spectateurs, ne laissant que peu de place pour des émotions véritablement ressenties et dues à des événements réels. Une autre possibilité est que ces stimulations affectives constantes activent en effet la sensibilité émotionnelle. Jusqu'ici, nous n'avons ni les concepts théoriques, ni les outils de recherches pour concevoir des études et examiner ces alternatives. Cependant, il est d'importance éducative d'étudier les effets de l'induction de commotions d'une façon similaire à la représentation de la 
violence. Un des résultats contraires pourrait être que les enfants grandissent en doutant de l'authenticité de beaucoup de ce qu'ils voient en ce qui concerne l'expression émotionnelle.

\section{L'impact des nouvelles technologies de communication sur l'expression émotionnelle et la régulation affective}

19 L'étude interculturelle entreprise par Scherer et al. (1986) a prouvé que la grande majorité des épisodes émotionnels intervient dans un contexte d'interaction sociale, produit par le comportement, souvent communicatif, d'autres personnes. Tandis que dans un passé pas si lointain la plupart des épisodes émotionnels se déroulaient dans des interactions de face-à-face, l'utilisation croissante des technologies modernes de communication (téléphones cellulaires, email, chats sur Internet) conduit à une proportion toujours croissante d'émotions interpersonnelles médiatisées. Cependant, il existe actuellement peu d'études détaillées sur la façon dont l'utilisation de cette technologie modifie la genèse, la régulation et la communication des états émotionnels.

Prenons l'exemple du téléphone portable. L'augmentation du nombre d'utilisateurs de la téléphonie mobile a été phénoménale et il y a peu de raisons de penser qu'elle diminuera dans un proche avenir. De plus, dans les pays industrialisés avec un niveau socioculturel élevé, beaucoup d'enfants et d'adolescents grandissent en pensant que c'est la chose la plus normale au monde que de posséder un téléphone mobile et de pouvoir atteindre (ou être atteint par) quiconque à tout moment. Tandis qu'il existe maintenant une certaine réglementation quant à l'utilisation des téléphones portatifs dans les espaces publics (par exemple, salles de concert, églises, etc.), la plupart des personnes semblent être tout à fait tolérantes par rapport à leur utilisation dans un grand nombre de contextes sociaux.

Comment est-ce que cette tendance en constante augmentation à utiliser les téléphones portatifs en public affectera-t-elle les émotions? Une conséquence évidente d'une telle utilisation est que la production des émotions dans ces espaces est susceptible d'augmenter. Beaucoup de nouvelles plaisantes ou désagréables, qui peuvent donner lieu à des éruptions immédiates d'émotions positives ou négatives, sont communiquées par téléphone. De même, l'utilisation croissante du téléphone pour toutes sortes de négociations et d'arrangements semble favoriser les démonstrations d'émotions en public. Ces comportements ont des conséquences intéressantes, notamment en ce qui concerne la régulation émotionnelle. En effet, dans beaucoup de sociétés, il a été d'usage de traiter les émotions comme une affaire privée, voire de décourager l'expression de l'émotion en public. Ce phénomène a déjà été décrit par $W$. Wundt dans son "Völkerpsychologie» de 1900 et popularisé sous le nom de display rules (règles de conduite) par Ekman et Friesen (1969). On peut se demander alors ce qu'il adviendra du recours au téléphone portable dans de telles sociétés, et en particulier quelles en seront les conséquences sur l'expression des émotions.

L'acceptation croissante du téléphone portable dans tous les contextes publics mènera probablement à un changement de ces display rules. Ainsi, les gens trouveront à l'avenir parfaitement normal que la personne assise à côté d'eux dans l'autobus ou à la table voisine au restaurant pleure de tristesse, crie de bonheur ou hurle de colère. Par ailleurs, les individus éprouvant ces émotions pourraient également trouver de plus en plus 
normal de les exprimer de façon toujours plus désinvolte en public. Après tout, si on ne s'inquiète pas de discuter de sujets plutôt personnels à haute voix dans les lieux publics, pourquoi se soucierait-on d'exprimer ouvertement ses émotions - qui font souvent partie intégrante d'une telle discussion. Un relâchement si général des contraintes émanant des règles de conduite peut mener à une augmentation de l'expressivité et, par conséquent, de l'importance de l'expression émotionnelle dans les interactions interpersonnelles conduites en public (et probablement aussi en privé). Et ceci d'autant plus si les médias continuent à encourager la production d'expressions exagérées, comme cela est déjà fréquent pour beaucoup d'émissions télévisées (par exemple, les jeux télévisés).

Un autre sujet d'étude est l'expression et l'expérience émotionnelles vécues tout en participant aux sessions de groupe sur l'Internet (par exemple, jeux vidéo interactifs, forums de discussion, etc.). La sociologue S. Turkle (1995) a intensivement étudié ces formes de communications électroniques (voir également Andersen, Holmquist et Jensen, 1993), principalement les jeux de rôle dans des domaines multi-utilisateurs. Une des particularités de ce type d'interactions est la possibilité offerte au participant de se redéfinir, de créer un persona virtuel souvent doté d'une personnalité ainsi que de dispositions affectives et comportementales différentes et, par là, de se mettre dans la peau de beaucoup de personnages différents. Ceci, combiné avec l'anonymat de cet environnement, mène souvent à des échanges fortement émotionnels et expressifs. Etonnamment, le contenu émotionnel est explicitement codé dans une forme de communication symbolique sous forme d'icônes affectifs. Sous une forme plus vocale, que le futur développement de ces domaines multi-utilisateurs offrira, on peut s'attendre à une expression affective encore plus forte.

24 L'analyse de S. Turkle sur les interactions ayant cours lors de forums de discussion prouve que l'intensité affective de ces échanges atteint des niveaux souvent tout à fait remarquables, exigeant dans certains cas l'application de règles de netiquette par des administrateurs ou par des coalitions d'utilisateurs. Dans ce contexte virtuel, l'intensité émotionnelle est parfois telle qu'elle peut effectivement engendrer des problèmes psychiques à long terme. Dans de nombreuses villes, les cliniques psychiatriques ont déjà établi des services de consultation pour la dépendance à Internet. Le problème peut non seulement être une dépendance qui rend le fonctionnement normal impossible, mais qui peut également induire une déréglementation de la vie émotionnelle (Young, 2004).

Comment les émotions virtuelles dans les jeux et les chats sur le net diffèrent-elles des émotions «normales»? S. Turkle (1995, p. 192) écrit: « En somme, les jeux de rôle dans des domaines multi-utilisateurs effacent les frontières entre l'individu et le jeu, l'individu et le rôle, l'individu et la simulation. » Etant donné le rôle important de l'individu (ces buts et valeurs actuels) dans l'émergence des émotions, on peut imaginer que dans un tel contexte, la genèse des émotions soit très complexe et potentiellement éloignée de la réalité. En particulier, non seulement les participants peuvent définir leur self, mais en plus, étant donné la nature fictive d'une grande partie de l'interaction, ils peuvent également définir certains critères d'évaluation, tels que leur capacité de contrôle ou leur pouvoir sur l'événement. S. Turkle (p. 189) cite un exemple : «j'aime jouer le rôle d'un héros, habituellement un avec des pouvoirs magiques... parfois je joue quelques parties réussies sur le net pour m'échauffer avant d'aller voir mon patron ». Ceci suggère que, contrairement à d'autres formes de jeux dans lesquelles les émotions demeurent limitées à la phase de jeu, l'identification élevée des joueurs virtuels avec leur identité imaginaire et leur capacité de manipuler leurs émotions peuvent induire une fusion entre l'affect 
réel et le virtuel. De cette façon, il peut y avoir une intégration des expériences émotionnelles de différentes origines et un débordement des émotions virtuelles dans la réalité.

Cette crainte se trouve renforcée par le récent et fulgurant développement technologique de ce domaine. Un article paru dans Le Temps («La victoire des elfes », par L. Chappez, 28 janvier 2006) relève: «Les jeux de rôle en ligne ne cessent de gagner des adeptes. Apologie du romanesque, ces mondes virtuels répondent au désir séculaire de s'évader du réel. » L'industrie vidéoludique a réussi à faire des MMORPG (massively multiplayer online role playing games ou jeux de rôle en ligne massivement multi-joueurs) un divertissement de masse. Un adepte témoigne: «... ce type de jeu engendre effectivement une dépendance comparable à une drogue. Vu le caractère immersif du jeu, et la vie sociale qui s'y développe, il est facile de sombrer dans un cercle vicieux : on sort de moins en moins, on perd le contacte avec ses connaissances, le monde réel semble de plus en plus ennuyeux et fade, donc on se replonge dans le monde virtuel... jusqu'à ne plus ressentir le moindre désir d'en sortir." Des auteurs ont toutefois proposé que ces jeux de rôle informatisés pouvaient aussi avoir des effets positifs. Ils pourraient être perçus comme une sorte de "laboratoire d'identité » aidant au développement du self (Turkle, 1995), comme un moyen de développer des capacités sociales et de créer des liens d'amitié basé sur un True Self subjectif (Bargh, McKenna \& Fitsimmons, 2002) ou encore comme un terrain efficace pour exercer rapidement sa sociabilité (Castronova, 2005). Pour le moment, ces suggestion restent spéculatives: il n'y a guère de données empiriques solides qui démontrent un transfert réussi des compétences acquises dans les MMORPGs sur les performances sociales réelles. En revanche, le témoignage cité ci-dessus évoque des dangers certains. Par exemple, la difficulté croissante de se libérer du monde virtuel pourrait amener une fusion entre réalité et fantaisie. De même, les émotions pourraient perdre leur potentiel adaptatif aux contingences de l'existence dans un monde peuplé d'humains, plutôt que d'avatars, et d'événements réels et irréversibles, cette perte ayant un effet certain sur le bien-être. La compétence émotionnelle la plus touchée pourrait être la capacité de régulation émotionnelle et de maîtrise (coping) : la tendance à avoir recours à la " pensée magique ", qui pourrait être renforcé par une immersion excessive dans les jeux en ligne, peut être considéré comme une stratégie très inadaptée. De fait, on observe une augmentation des cas de dépendance sévère aux MMORPGs avec les conséquences psychosociales graves qui en découlent; comme mentionné ci-dessus, il existe actuellement un nombre important de consultations psychologiques et psychiatriques qui traitent ces dépendances. En parallèle, des tentatives de réglementation de l'accès excessif à ces jeux sont en cours dans certains pays (en Chine, par exemple). Malheureusement, comme pour les effets positifs supposés, peu de recherches systématiques ont été jusqu'ici menées pour confirmer les troubles socioaffectifs engendrés par ce genre de dépendance.

La discussion précédente suggère que l'utilisation de la technologie moderne de communication peut promouvoir une expression émotionnelle plus libre ou même désinhibée aussi bien qu'une déconnexion du vécu affectif de la réalité. Un tel développement irait à l'encontre de l'augmentation du contrôle de l'affect que N. Elias (1973) a considéré comme la garantie du processus de civilisation. Si tel était le cas, on pourrait s'attendre à des conséquences non seulement sur les interactions sociales (par exemple, le retour aux formes médiévales plus rudes d'expression affective " débridée ", qui étaient communes avant que les manières courtoises exigent un contrôle de l'affect), 
mais également sur la régulation des affects. Par exemple, si on adoptait l'hypothèse de catharsis, l'expression désinhibée des affects devrait en principe avoir un effet positif de «nettoyage ». Inversement, si on adoptait l'hypothèse de rétroaction proprioceptive (voir McIntosh et al., 1997), elle pourrait réellement intensifier l'émotion (voir Scherer, 2001, pour une évaluation comparative de ces deux alternatives et une tentative d'intégration). Pour conclure, si en effet il devait y avoir une tendance à exprimer et à expérimenter plus intensément les émotions suite à l'utilisation massive des technologies de communication mentionnées précédemment, ceci constituerait un sujet de recherche d'importance fondamentale pour les sciences sociales.

\section{BIBLIOGRAPHIE}

Anderson C. A. \& Bushman B. J., « The Effects of Media Violence on Society », in Science, vol. 295, $\mathrm{n}^{\circ} 5564,2002$, p. 2377-2379.

Andersen P. B., Holmqvist B. \& Jensen J. F. (eds.), The computer as medium, New York, Cambridge University Press, 1993.

Bargh J. A., McKenna K. Y. A. \& Fitsimmons G. M. « Can You See the Real Me ? Activation and expression of the 'True Self' on the Internet ", Journal of Social Issues, vol. 58, n' 1, 2002, p. 38-48.

Baudonnière P. M., Le mimétisme et l'imitation, Paris, Flammarion, 1997.

Bischof-Köhler D., « The development of empathy in infants », in M. E. Lamb, \& H. Keller (eds.), Infant development : Perspectives from German-speaking countries, Hillsdale, Erlbaum, 1991, p. 245-273.

Borg I., Staufenbiel Th. \& Scherer K. R., « On the symbolic basis of shame », in K. R. Scherer (ed.), Facets of emotion : Recent research, Hillsdale, Erlbaum, 1988, p. 79-98.

Cantor J. \& Nathanson A. I., " Children's fright reactions to television news », Journal of Communication, vol. 46, $\mathrm{n}^{\circ} 4,1996$, p. 139-152.

Castronova E., Synthetic Worlds : The Business and Culture of Online Games, Chicago, University of Chicago Press, 2005.

Delumeau J., La peur en Occident, Paris, Fayard, 1978.

Ekman P. \& Friesen W. V., « The repertoire of nonverbal behavior : Categories, origins, usage, and coding ", Semiotica, $\mathrm{n}^{\circ} 1,1969$, p. 49-98.

Elias N., La civilisation des mœurs, Paris, Calmann-Levy, 1973 (1 1 ère édition allemande, 1939).

Ellsworth P. C. \& Scherer K. R., « Appraisal processes in emotion », in R. J. Davidson, H. Goldsmith, \& K. R. Scherer (eds.), Handbook of the Affective Sciences, New York and Oxford, Oxford University Press, 2003, p. 572-595.

Freedman J. L., Media Violence and its Effect on Aggression : Assessing the Scientific Evidence, Toronto, University of Toronto Press, 2002.

Frijda N. H., The emotions, Cambridge, Cambridge University Press, 1986. 
Gaulejac V. de, Les sources de la honte, Paris, Desclée de Brouwer, 1996.

Gleich U. \& Groebel J., « Die emotionale Qualität von (Fernseh-)Nachrichten : Zuschauerinteresse und Wirkung ", Media Perspektiven, $n^{\circ}$ 9, 1993, p. 445-449.

Goleman D., Emotional Intelligence, New York, Bantam Books, 1995.

Hatfield E., Cacioppo J. T. \& Rapson R. L., « Emotional contagion », Current Directions in Psychological Science, vol. 2, n 3, 1993, p. 96-99.

Johnston W. M. \& Davey G. C. L., « The psychological impact of negative TV news bulletins : The catastrophizing of personal worries ", British Journal of Psychology, vol. 88, nº 1, 1997, p. 85-91.

Lakin J. L., Jefferis V. E., Cheng C. M. \& Chartrand T. L, « The Chameleon Effect as Social Glue : Evidence for the Evolutionary Significance of Nonconscious Mimicry ", Journal of Nonverbal Behavior, $\mathrm{n}^{\circ}$ 27, 2003, p. 145-162.

Lepenies W., Melancholie und Gesellschaft, Frankfurt, Suhrkamp, 1969.

Lewis M., Shame: The exposed self, New York, Free Press, 1992.

Lynd H., Shame and the search for identity, New York, Harcourt Brace, 1970.

McIntosh D. N., Zajonc R. B., Vig P. S. \& Emerick S. W., « Facial movement, breathing, temperature, and affect : Implications of the vascular theory of emotional efference ", Cognition and Emotion, vol. 11, n 2, 1997, p. 171-195.

Piers G. \& Singer M. B., Shame and guilt, New York, Norton, 1971.

Roseman I. J. \& Smith C. A., « Appraisal theory : Overview, assumptions, varieties, controversies ", in K. R. Scherer, A. Schorr, \& T. Johnstone (eds.), Appraisal processes in emotion : Theory, Methods, Research, New York and Oxford, Oxford University Press, 2001, p. 3-19.

Scheff T. J., "Socialization of emotions : Pride and shame as causal agents », in T. D. Kemper (ed.), Research agendas in the sociology of emotions. SUNY series in the sociology of emotions, Albany, State University of New York Press, 1990, p. 281-304.

Scherer K. R., " On the symbolic functions of vocal affect expression ", Journal of Language and Social Psychology, nº 7, 1988, p. 79-100.

- « The role of culture in emotion-antecedent appraisal ", Journal of Personality and Social Psychology, nº 73, 1997, p. 902-922.

- «Emotionsprozesse im Medienkontext : Forschungsillustrationen und Zukunftsperspektiven », Medienpsychologie, vol. 10, $\mathrm{n}^{\circ}$ 4, 1998, p. 276-293.

- « The nature and study of appraisal : A review of the issues ", in K. R. Scherer, A. Schorr, \& T. Johnstone (eds.), Appraisal processes in emotion : Theory, Methods, Research, New York and Oxford, Oxford University Press, 2001, p. 369-391.

- « Emotional expression is subject to social and technological change : Extrapolating to the future », Social Science Information, vol. 40, n 1, 2002, p. 125-151.

Scherer K. R., Wallbott H. G. \& Summerfield A. B. (eds.), Experiencing emotion : A crosscultural study, Cambridge, Cambridge University Press, 1986.

Scherer K. R. \& Zentner M., « Emotional effects of music : production rules », in P. N. Juslin, \& J. A. Sloboda (eds), Music and emotion: Theory and research, Oxford, Oxford University Press, 2001, p. 361-392. 
Schorr A., « Realitätsmanagement beim Fernsehkonsum. Ein Beitrag zur Wirkung von RealityTV-Sendungen auf das emotionale Befinden », Medienpsychologie :-Zeitschrift-für-Individual-andMassenkommunikation, vol. 7, n 3, 1995, p. 184-204.

Stearns C. Z. \& Stearns P. N., Anger: The Struggle for Emotional Control in America's History, Chicago, University of Chicago Press, 1986.

- Emotion and social change: Toward a new psychohistory, New York, Holmes \& Meier, 1988.

Turkle S., Life on the screen : Identity in the age of the Internet, New York, Simon \& Schuster, 1995.

Wallbott H. G. \& Scherer K. R., «The antecedents of emotional experience », in K. R. Scherer, H. G. Wallbott, \& A.B. Summerfield (eds.), Experiencing emotion : A cross-cultural study, Cambridge, Cambridge University Press, 1986, p. 69-83.

- «Cultural determinants in experiencing shame and guilt», in J. P. Tangney, \& K. W. Fischer (eds.), Self-conscious emotions: The psychology of shame, guilt, embarrassment, and pride, New York, Guilford Press, 1995, p. 465-487.

Winterhoff-Spurk P., « Violence in TV news : The cultivation of emotions », in P. WinterhoffSpurk, \& T. H. A. van der Voort (Hrsg.), New horizons in media psychology. Research cooperation and projects in Europe, Opladen, Westdeutscher Verlag, 1997, p. 105-115.

Wundt W., Völkerpsychologie. Eine Untersuchung der Entwicklungsgesetze von Sprache, Mythos und Sitte (Band I. Die Sprache), Leipzig, Kröner, 1900.

Young K. S., «Internet Addiction : A New Clinical Phenomenon and Its Consequences », American Behavioral Scientist, vol. 48, $\mathrm{n}^{\circ}$ 4, 2004, p. 402-415.

\section{NOTES}

1. Cette contribution résume et développe des idées présentées dans un numéro spécial sur le futur des émotions dans "Social Science Information/Informations sur les Sciences Sociales » (Scherer, 2002). Je tiens à remercier Rachel Baeriswyl-Cottin et Patricia Martorell pour l'aide précieuse qu'elles ont apportée à la rédaction et à la mise en forme de ce manuscrit.

\section{AUTEUR}

\section{KLAUS SCHERER}

Section de psychologie

Pôle de Recherche National Suisse en Sciences Affectives

Université de Genève

klaus.scherer@pse.unige.ch 